\title{
Globe
}

Revue internationale d'études québécoises

\section{Daniel Jacques : Nationalité et modernité, Montréal, Boréal, 1998, 268 p.}

\section{Jocelyne Couture}

Volume 3, numéro 1, 2000

URI : https://id.erudit.org/iderudit/1000573ar

DOI : https://doi.org/10.7202/1000573ar

Aller au sommaire du numéro

Éditeur(s)

Globe, Revue internationale d'études québécoises

ISSN

1481-5869 (imprimé)

1923-8231 (numérique)

Découvrir la revue

Citer ce compte rendu

Couture, J. (2000). Compte rendu de [Daniel Jacques : Nationalité et modernité, Montréal, Boréal, 1998, 268 p.] Globe, 3(1), 165-169.

https://doi.org/10.7202/1000573ar d'utilisation que vous pouvez consulter en ligne.

https://apropos.erudit.org/fr/usagers/politique-dutilisation/ 


\section{RECENSIONS}

avant de culminer dans le messianisme du dernier quart de siècle. Plutôt que de chicaner sur ses carences historiennes, je crois qu'il faut d'abord saluer l'originalité d'une telle entreprise et espérer que semblable lecture soit réalisée de L'Avenir ou de La Vérité.

Lucie Robert

Université du Québec à Montréal

\section{Daniel Jacques \\ Nationalité et modernité \\ Montréal, Boréal, 1998, 268p.}

La nation, qui représente pourtant la forme d'association politique la plus répandue à notre époque, a cessé d'être un idéal politique. Cet état de choses, analysé par Daniel Jacques, n'est selon lui qu'une manifestation, parmi bien d'autres, d'un mal caractéristique de ce siècle : la confusion des allégeances. Incapables de lier de façon cohérente les obligations résultant de leur appartenance à une communauté et certaines nécessités politiques auxquelles ils font face, nos contemporains auraient choisi de faire prévaloir les secondes, sapant ainsi les assises morales de la nation et sectionnant du même coup le lien qui unit le moral et le politique. Pourtant la pluralité des allégeances, marque distinctive de la vie dans une société démocratique, ne nous condamne pas à une telle confusion car, selon l'auteur, elle trouve sa source ainsi que son principe intégrateur au sein même des idéaux de la modernité qui ont façonné l'évolution des sociétés occidentales. Loin de vouloir déplorer la pluralité des allégeances, l'auteur veut au contraire défendre la thèse selon laquelle la nation peut en constituer une synthèse cohérente et qu'elle est à cet égard, "la condition de réalisation de l'idéal moderne» (p. 19).

La question de la légitimité politique de la nation nous renvoie donc, soutient l'auteur, à l'histoire de la pensée politique et aux débats philosophiques au cours desquels se sont précisés les idéaux et les exigences, parfois conflictuels, de la modernité. Dans un premier chapitre, intitulé «L'esprit d'égalité», l'auteur soutient, en s'appuyant 


\section{REVUE INTERNATIONALE D'ÉTUDES QUÉBÉCOISES}

sur une analyse de la pensée politique de Platon, Thomas d'Aquin, Pascal, Hobbes, Locke, Machiavel, Montesquieu, Rousseau et Kant, qu'à l'origine de la modernité politique se trouve une profonde révolution morale. Par-delà leurs profondes divergences, les Modernes ont en commun, dit-il, de rejeter l'inégalité entre les personnes que les Anciens placent au cœur de la vie philosophique. C'est pourquoi il serait devenu si évident aux yeux des Modernes que tous ont le droit de participer à la définition de l'autorité commune et que chacun a le droit de définir sa vie comme il l'entend. Ainsi les idéaux d'autonomie, de liberté, d'authenticité et de démocratie, si chers à nos contemporains, prennent leur source dans le principe d'égalité qui fonde la modernité.

Ces idéaux de la modernité, cependant, ont donné lieu à une pluralité de revendications qui n'ont pas toujours réussi à rendre à chacun d'eux la place qui lui revenait. C'est ce que montrent les deuxième et troisième chapitres de Nationalité et modernité, où ce sont les conceptions historiques de la nation qui, cette fois, devraient nous permettre de mieux comprendre la modernité. Le second chapitre, intitulé «La société des individus», porte sur le libéralisme de Benjamin Constant et de Tocqueville. L'auteur montre comment, dans le cadre d'une société de droit qui consacre la priorité de l'universel des droits de la personne sur la différence entre les peuples, la nation se réduit à un lieu pour réaliser le principe de souveraineté populaire. Le désaveu (Constant) ou la menace de dissolution (Tocqueville) des appartenances particulières au profit d'un patriotisme civique fondé sur le simple partage de la liberté expose ainsi la société libérale à l'uniformisation, à l'effritement des bases de l'autonomie des personnes et, finalement, à l'érosion du politique comme lieu de reconnaissance mutuelle.

Dans son troisième chapitre, aLa révolution nationales, l'auteur examine la revendication inverse qui consiste à accorder à la nation, à la culture et à l'histoire particulières une priorité sur l'universel. Il y est question de cla révolution conservatrices instiguée en Allemagne au début du siècle par Oscar Spengler, Ernst Jünger, Hans Freyer et à laquelle contribuent aussi Carl Schmitt et Heidegger. «Révolution» parce que, contrairement au retour au passé et à la tradition 


\section{RECENSIONS}

préconisé par Joseph de Maistre par exemple, elle suppose, au nom de la nation, un dépassement radical de toutes les traditions et vise à l'établissement d'une nouvelle figure de la communauté. L'auteur insiste sur le fait que ce projet révolutionnaire, qui a conduit à la pire catastrophe de notre siècle, demeure unique dans l'histoire de la pensée politique. Sans vouloir lui imputer la responsabilité de cette tragique dérive, l'auteur reconnaît cependant l'influence déterminante de la pensée de Nietzsche sur les principaux protagonistes de la révolution conservatrice qu'il analyse comme un refus de la modernité et du libéralisme. La révolution conservatrice et la pensée de Nietzsche seraient-elles pour autant hors de la modernité? Un trait distinctif de la modernité, nous dit l'auteur, est son ambivalence vis-à-vis d'elle-même. «Depuis Rousseau le soupçon est lâché que la société des individus ne saurait devenir une véritable communauté politique. La droite, tout comme la gauche, a su, à ce jour, récupérer pour elle-même ce sentiment d'un déclin général du vivre ensemble* (p. 171).

À partir de la seconde moitié de l'ouvrage, qui entame la question proprement dite de la légitimité de la nation, l'auteur se tourne vers les aspects du contexte contemporain que doit prendre en compte une défense, prudente, souligne-t-il, de la nation. Au début du quatrième chapitre, intitulé «Les lieux de la reconnaissance», l'auteur affirme que c'est à partir d'un idéal moral et d'une catastrophe historique que nous devons entreprendre de penser la légitimité politique de la nation. D'une catastrophe historique, parce que, dit-il, *[c]e qui affecte toute défense de la nation et rend difficile son introduction dans le champ de la pensée politique, c'est la condamnation morale qui pèse sur elle» depuis l'Holocauste (p. 174). Pour ce qui est de l'idéal moral, nous avons, dit l'auteur, un idéal d'autonomie, mais, poursuit-il, «cette conception de la liberté n'est cependant devenue pour nous un fondement [...] que sous l'évidence d'un mal radical qui s'est offert dans l'événement» (p. 175).

L'auteur précise d'abord le sens du politique, caractérisé selon lui par l'institution d'un ordre commun qui implique la distinction entre le commun du particulier, la clôture du champ social, la délimitation du territoire et la définition d'un régime, particulier ou universel, de 
reconnaissance. Sur ce dernier point, et en tant qu'exemples de positions extrêmes à éviter, l'auteur examine les propositions d'Alexandre Kojève pour un État universel et de Léo Strauss pour une société fermée et hiérarchique. L'auteur avance que la mise en commun qui fonde le politique est une question de justice et que toute politique de la nation vise à protéger la communauté de sens fondée sur la reconnaissance réciproque et requise par la mise en commun originaire tout autant que par la liberté démocratique. La distinction libérale qui relègue le sens dans la sphère privée et la justice dans la sphère publique du politique n'a donc rien à faire ici de même que les principes, individualistes selon l'auteur, qui dissocient les quêtes d'identité, de liberté et de justice. La conception de la nation qui se dégage de cette discussion est celle de la nation culturelle, historique et politique qui s'oppose tout autant à la nation civique qu'à la nation ethnique et qui récuse de la même manière les conceptions cosmopolitique et patriarcale de la société. L'auteur insiste cependant, comme plusieurs autres l'ont fait avant lui, sur le fait que la nation ainsi conçue, tout en étant d'inspiration communautarienne, demeure enracinée dans une préoccupation universelle. L'auteur aborde finalement la question de la clôture de l'espace politique, c'est-à-dire de la délimitation du territoire national. L'épineuse question sera résolue par un appel, utopique de l'avis même de l'auteur, à la reconnaissance identitaire et au respect mutuel que s'accordent les individus concernés.

Le dernier chapitre, intitulé "Politique de la nation", aurait tout aussi bien pu servir de conclusion à l'ouvrage. L'auteur y justifie son approche en soulignant qu'il y a malentendu à penser la nation en termes de résistance à l'universel ou au cosmopolitisme plutôt qu'en termes des idéaux de liberté et d'authenticité des modernes. Il y fait aussi l'éloge de la prudence qui doit présider à la défense de la nation, prudence qui, selon lui, doit nous inciter à éviter les extrêmes, à rechercher plutôt un équilibre entre toutes les tendances de la modernité et à tenir compte du contexte historique dans lequel $s$ 'inscrit cette défense. Pour sa part, l'auteur affirme que c'est dans le cadre particulier d'un libéralisme sans partage que s'inscrit sa propre défense de la nation. C'est d'ailleurs ce contextualisme qui lui permet d'affirmer que la nation, telle qu'il l'entend, réalise une synthèse 
pratique, et non pas théorique, des idéaux de la modernité. Cette synthèse est aussi pratique au sens où toute philosophie politique devrait l'être, pourrions-nous ajouter, c'est-à-dire au sens où elle contribue à faire apercevoir des solutions de rechange, à la fois faisables et moralement acceptables, pour des situations qui font présentement obstacle à l'émancipation des personnes et des sociétés. Tel est l'engagement philosophique qui se manifeste tout au long de cet ouvrage sans jamais porter ombrage à sa rigueur et à la perspicacité des analyses qu'il contient.

Jocelyne Couture

Université du Québec à Montréal

\section{Henri Lamoureux}

Les Dérives de la démocratie.

Questions à la société civile québécoise

Montréal, VLB éditeur, 1999, 159p.

Cet ouvrage s'inscrit dans un débat animé sur la transformation de la pratique sociale qui affecte le mouvement communautaire depuis quelques années. L'essayiste et romancier Henri Lamoureux, dans un style polémique qui n'est pas dépourvu de dérives verbales, tente de mettre au jour certaines tendances à l'œuvre au sein de la société civile qui détoumeraient cette dernière de son objectif d'émancipation de la population. Notons ici que le terme «société civile» est utilisé dans l'ouvrage dans son sens le plus idéaliste, entre autres comme uobstacle aux effets pervers d'une économie de marché» (p.12).

L'auteur identifie trois tendances au sein de la société civile qui menaceraient son idéal de démocratie et d'humanisme. La première est constituée du corporatisme des pratiques sociales (ou encore le néo-corporatisme selon les passages) qui «atteste l'importance croissante d'une vision utilitaire et économiste de l'action communautaire qui, faute d'avoir le courage d'adopter une 